СОЛНЕЧНАЯ АКТИВНОСТЬ ЗА ПОСЛЕДНИЕ МИЛЛИОНЫ ЛЕТ ПО ДАННЫМ О ДОЛГОЖИВУЩИХ РАДИОНУКЛИДАХ В ЛУННЫХ ПОРОДАХ

Васильев Г.И. ${ }^{1}$, Дергачёв В.А. ${ }^{1}$, Константинов А.Н. ${ }^{2}$, Кудрявцев И.В. ${ }^{1}$, Остряков В.М. ${ }^{2}$, Павлов А.К. ${ }^{1}$, Фролов Д.А. ${ }^{1}$

${ }^{1}$ Физико-технический институт им. А.Ф. Иоффе РАН, Санкт-Петербург, Россия

${ }^{2}$ Санкт-Петербургский политехнический университет Петра Великого,

Санкт-Петербург, Россия

\title{
ESTIMATES OF SOLAR ACTIVITY WITHIN THE LAST MILLIONS OF YEARS BASED ON RADIONUCLIDE MEASUREMENTS IN LUNAR ROCKS
}

\author{
Vasilyev G.I. ${ }^{1}$, Dergachev V.A. ${ }^{1}$, Konstantinov A.N. ${ }^{2}$, Koudriavtsev I.V. ${ }^{1}$, \\ Ostryakov V.M. ${ }^{2}$, Pavlov A.K. ${ }^{1}$, Frolov D.A. ${ }^{1}$ \\ ${ }^{1}$ Ioffe Institute, St-Petersburg, Russia \\ ${ }^{2}$ Peter the Great St-Petersburg Polytechnic University, St-Petersburg, Russia
}

We have estimated the values of solar modulation parameter $\Phi$ on several time scales: $20 \mathrm{kyr}, 2 \mathrm{Myr}$ and $6 \mathrm{Myr}$, using nuclear code GEANT4.10 and experimental data on ${ }^{14} \mathrm{C},{ }^{26} \mathrm{Al}$ and ${ }^{10}$ Be activity in Apollo 15 drill core. We report the inaccuracies of direct GEANT4 application to the problem and propose the calibration procedure based on terrestrial radiocarbon data to correct the calculations of GCR interaction with the Moon surface. Those corrections are justified by recent comparisons of the Lunar Neutron Probe data with neutron fluxes calculated via GEANT4. The resulted mean $\Phi$ over the last $2 \mathrm{kyr}$ and $2 \mathrm{Myr}$ are in good agreement with modern understanding of levels of solar activity, however, the 6 Myr estimate suggest a strong additional source of high-energy GCR particles.

DOI: 10.31725/0552-5829-2021-71-74

В данной работе проведено моделирование образования космогенных радионуклидов в Лунном грунте с использованием GEANT4.10. Методика расчетов предполагает вычисление функции выхода изотопа в образце на одну падающую частицу с фиксированной энергией и последующую свертку этой функции со спектром падающих частиц (подробнее см. [1]). В расчетах использовалась параметризация спектра ГКЛ $\mathrm{J}_{\mathrm{US05}}(\mathrm{E})$ и формула модуляции из [2]. Поскольку Луна является, по сути, интегральным детектором КЛ, то, используя экспериментально измеренные профили [3-5], возможно восстановить средний модуляционный потенциал Ф на протяжении $\sim 4.5$ периодов полураспада конкретного радионуклида. Модуляционный потенциал Ф напрямую связан с уровнем солнечной активности.

Аналогичные расчеты для земных архивов радиоуглерода позволили восстановить временной ход Ф на протяжении последних 19000 лет [6, 7]. Применив эти данные к расчетам активности ${ }^{14} \mathrm{C}\left(\mathrm{T}_{1 / 2} \sim 6\right.$ тыс. лет) на Луне, 
мы получили рассогласование расчетов с экспериментальными данными, которое, однако, можно устранить введением линейного коэффициента $\mathrm{Y}_{0} \sim 0.6$ (см. рис. 1). Обосновать введение такого коэффициента можно завышенным количеством вторичных нейтронов в GEANT4.10. Так, в работе [8] показано отличие расчетной и экспериментальной плотности вторичных нейтронов, причем соответствие эксперименту также достигается умножением на тот же линейный фактор. Значение $\Phi=360 \mathrm{MB}$, полученное затем с использованием такой поправки, согласуется со усредненным за последние 20 тыс. лет $\Phi(\mathrm{t})$ по данным о земной атмосфере. Проведя таким образом калибровку GEANT4.10 для лунного грунта, мы затем применяем аналогичную поправку для изотопов ${ }^{26} \mathrm{Al}$ и ${ }^{10} \mathrm{Be}$.

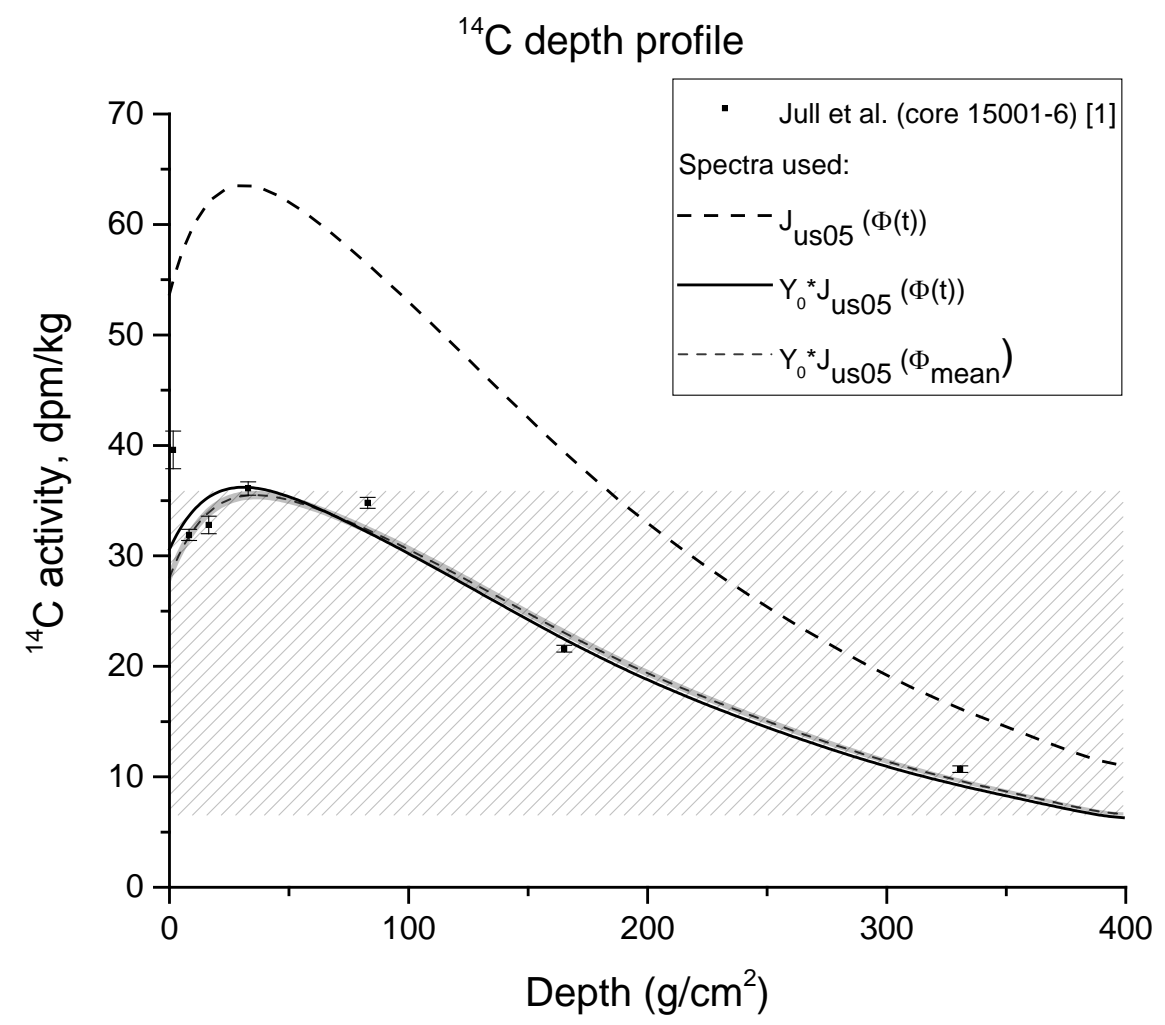

Рис. 1. Глубинные профили активности ${ }^{14} \mathrm{C}$

На рис. 2 приведены результаты для ${ }^{26} \mathrm{Al}$ ( $\mathrm{T}_{1 / 2} \sim 720$ тыс. лет). Видно, что использование поправки $\mathrm{Y}_{0}$ позволяет лучше описать экспериментальную кривую. Для сравнения также приведен результат из работы [9], в которой, по сути, был неявным образом применен такой же коэффициент, т.к. авторы постулировали время облучения образца 1 млн. лет. Наша оценка $\Phi=480$ МВ согласуется с полученным этими авторами значением $\Phi=496 \mathrm{MB}$ и соответствует умеренному среднему уровню солнечной активности за последние 3 млн. лет. 


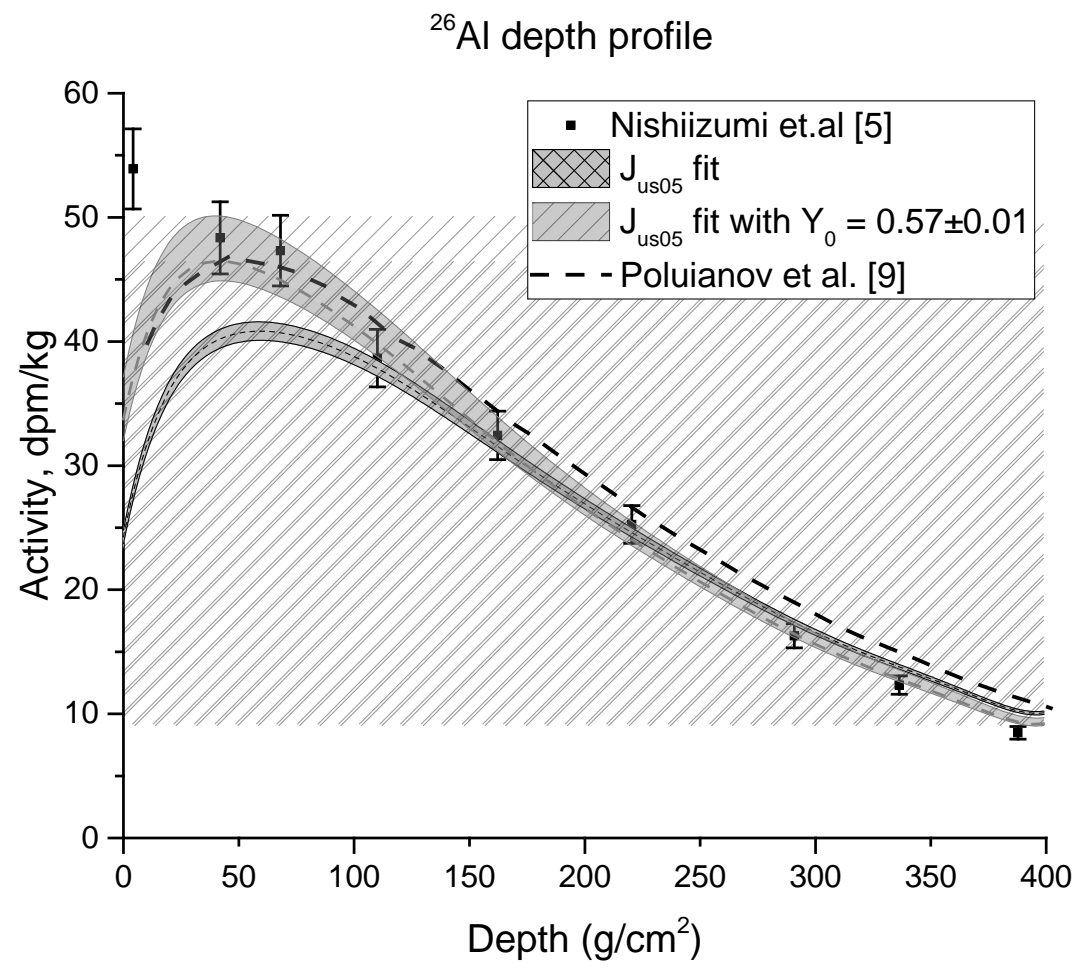

Рис. 2. Глубинные профили активности ${ }^{26} \mathrm{Al}$

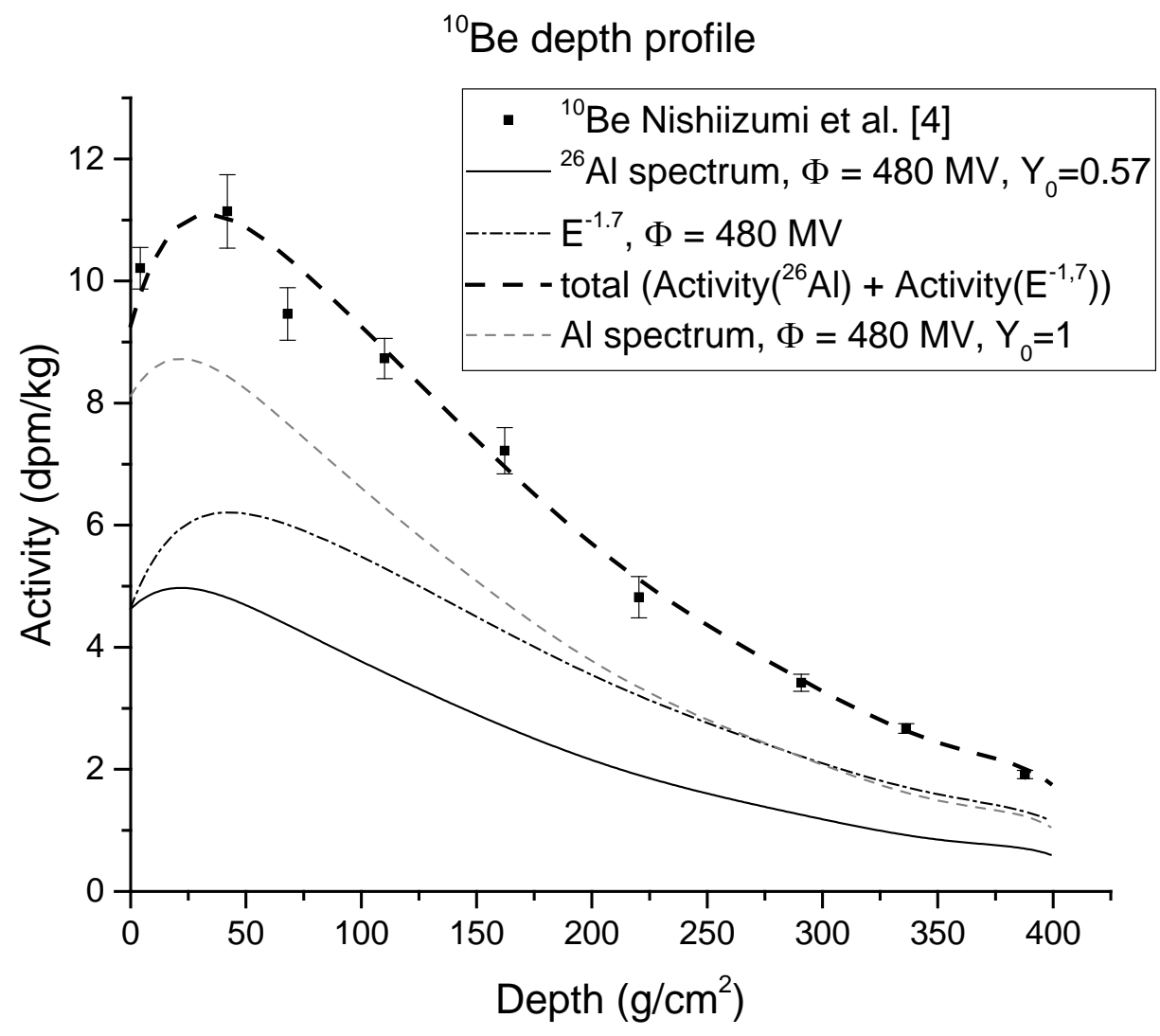

Рис. 3. Глубинные профили активности ${ }^{10} \mathrm{Be}$ 
В случае ${ }^{10}$ Ве $\left(\mathrm{T}_{1 / 2} \sim 1.4\right.$ млн. лет), приняв средние параметры спектра ГКЛ аналогичными оценкам по ${ }^{26} \mathrm{Al}$ и вычислив профиль ${ }^{10} \mathrm{Be}$ с этими параметрами, легко видеть, что экспериментальные данные невозможно объяснить без привлечения дополнительного источника жестких космических лучей, источником которых могла бы быть, например, близкая Сверхновая [10].

\section{Литература}

1. Pavlov A.K. et al. // Journal of Atmospheric and Solar-Terrestrial Physics, 2017, 164, 308.

2. Herbst K. et al. // Journal of Geophysical Research, 2010, 115, 1.

3. Jull A.J.T. et al. // Geochimica et Cosmochimica Acta, 1998, 62, 3025.

4. Nishiizumi K. et al. // Earth and Planetary Science Letters, 1984, 70, 157.

5. Nishiizumi K. et al. // Earth and Planetary Science Letters, 1984, 70, 164.

6. Kudryavtsev I.V., V.A. Dergachev // Geomagnetism and Aeronomy, 2019, 59, 1099.

7. Кудрявиев И.В., В.А. Дергачев // Сборник трудов ССЗФ, 2020, 185.

8. Mesick K.E. et al. // Earth and Space Science, 2018, 5, 324.

9. Poluianov S., G.A. Kovaltsov, I.G. Usoskin // Astronomy and Astrophysics, 2018, 618, 1. 10. Wallner A. et al. // Nature, 2016, 532, 69. 